\title{
Peripheral nerve sonography, a novel technique for improving the diagnosis of Hansen's neuropathy
}

\author{
Madhu Nagappa ${ }^{\mathrm{a}}$, Leo H. Visser ${ }^{\mathrm{b}}$ \& Lokesh Bathala ${ }^{\mathrm{c}}$ \\ ${ }^{\mathrm{a}}$ Department of Neurology, National Institute of Mental Health and \\ Neurosciences, Bangalore, 560029, India \\ ${ }^{\mathrm{b}}$ Department of Neurology and Clinical Neurophysiology, \\ Elisabeth-Tweesteden Hospital, Tilburg, The Netherlands \\ ${ }^{\mathrm{c}}$ Lead Consultant Neurologist, Aster CMI Hospital, Hebbal, Bangalore, \\ 560092, India
}

Submitted and accepted 18 August 2021

Leprosy is one of the oldest diseases known to mankind, and the first disease whose etiopathogenesis was linked to a bacterium. Despite efforts to eliminate leprosy through the worldwide leprosy elimination campaign and the enhanced global strategy, it continues to prevail in endemic areas with continued detection of newly infected cases. Although the new case incidence of leprosy has decreased quite tremendously, the level of Grade 2 disability, due to peripheral nerve damage which involves considerable clinical deficits and deformity, has remained unchanged. ${ }^{1}$ Early diagnosis and treatment of peripheral neuropathy is essential to prevent stigmatizing deformities and disabilities in leprosy. Diagnosis of leprosy reactions and underlying nerve injury would help to reverse the damage by prompt treatment.

Dysfunction of peripheral nerves, the primary targets of Mycobacterium leprae, is the cause of deformity and disfigurement, and the social stigma and ostracism that follow. The spectrum of neuropathy is governed by the host immune response and ranges on a continuum from tuberculoid to lepromatous leprosy. Pure neuritic leprosy, silent neuropathy, leprous ganglionitis and acute neuritis during lepra reactions may also occur. ${ }^{2}$

Traditionally, the diagnosis of leprosy relies on the presence of at least one of the three cardinal signs which are hypo-aesthetic skin lesions, thickened nerves and acid-fast bacilli in skin smears. While each of these cardinal signs individually has high sensitivity and specificity, errors arise when they are not correctly identified due to incomplete, inadequate or superficial clinical examination. Enlarged nerves have a limited number of differential diagnoses which include Charcot-Marie-Tooth disease, chronic inflammatory demyelinating polyradiculoneuropathy (CIDP), amyloid neuropathy and neurofibromatosis, all of which are quite rare. Among these conditions, leprosy is the only disorder where the identification of thickened nerves is 'central' to the diagnosis. The presence of tender, nodular thickened nerves

Correspondence to: Professor Dr. Leo H. Visser, Department of Neurology and Clinical Neurophysiology, Elisabeth-Tweesteden Hospital, Tilburg, The Netherlands (e-mail: lh.visser@etz.nl) 
in the presence of relatively preserved function is considered to be sine qua non of leprosy. At the tuberculoid end of the spectrum, nerve enlargement occurs because of granulomatous inflammation, while at the lepromatous end, infiltration of nerve fascicles with or without caseous necrosis underlies nerve enlargement. ${ }^{2}$

\section{Superiority of ultrasound above clinical palpation to detect nerve thickening}

The ability to detect these pathologically thickened nerves depends upon the experience, expertise and thoroughness of examination. Nerve thickening may be missed by casual or incomplete examination and vice-versa, normal nerves may be labelled as thickened by the less experienced clinicians and para-medical staff, leading to falsely-low or falsely-high detection rates. Gupte et al. reported that the inter-observer agreement among three senior examiners for the presence of skin patch, thickened nerves and sensory deficit was fair with a kappa of 0.7. ${ }^{3}$ In another study, the inter-observer agreement between a leprologist, students and other staff for the detection of thickened ulnar and popliteal nerves was at best modest with kappa values ranging from 0.4 to $0.6 .{ }^{4}$ Furthermore, palpation of commonly involved nerves to assess the nerve thickening, for example the median nerve, can be cumbersome due to the anatomical location of the nerve. Thus, the reliability of clinical examination is far from satisfactory. Longitudinal comparison of change in nerve thickness post-treatment cannot be carried out only with clinical data. Besides, other structures such as thrombosed veins may be sometimes clinically mistaken for thickened nerves.

High-resolution ultrasound (HRUS) of peripheral nerves can be used to study the morphology of the peripheral nerves along their entire length, except very proximally where they are deeply located. This is a clear advantage over clinical examination where enlargement of only those nerves that are located superficially can be detected, particularly as they traverse the bony prominences or in the periphery of a cutaneous lesion. ${ }^{5}$ HRUS provides an objective record of enlarged nerves based on established population norms and cut-off values.

Jain et al. reported that the kappa for identification of thickened nerves by clinical examination vis-à-vis HRUS was $0.3 .{ }^{6}$ Recently, Sreejith et al. also reported a lack of good agreement between clinical and sonographic examination for the detection of thickened nerves with a kappa of -0.04 for the median nerve to 0.215 for the common peroneal nerve. ${ }^{7}$ These data clearly indicate clinical examination alone is inadequate and HRUS identifies a greater number of patients with abnormal nerves even in the presence of normal electrophysiological testing. ${ }^{8}$ Sonographically enlarged nerves correlate with clinical and electrophysiological abnormalities. ${ }^{7,9}$ Being non-invasive and well tolerated by the patients, HRUS can be used to identify 'silent' neuropathy in patients with isolated cutaneous manifestations and normal neurological examination, as well as to screen household contacts. With technological advances, smaller ultrasound machines are available which can be used at the point-of-care. ${ }^{10}$ Paramedical field workers can probably be trained to identify thickened nerves more reliably by HRUS than by clinical examination; besides the ultra-sound data can be verified subsequently by an expert or in real-time using tele-medicine services.

\section{Specific localisation of nerve enlargement in leprosy and morphological characteristics}

Nerve enlargement in leprosy is characteristically fusiform and occurs a few centimetres proximal to the fibro-osseous canals such as the ulnar sulcus or carpal tunnel. ${ }^{9,11-13}$ This distinguishes leprosy from the more common entrapment neuropathies as well as from other neuropathies with enlarged nerves such as Charcot-Marie-Tooth disease and CIDP. ${ }^{5,12}$ Nerve 
enlargement in leprosy is typically asymmetrical and may involve a single nerve, especially in paucibacillary cases. The degree of enlargement has been shown to correlate with motor weakness. ${ }^{6,9}$ The individual fascicles within the nerve are also enlarged in leprosy. Increased thickness of the epineurium is often detected even in asymptomatic subjects, which correlates with the enlarged cross-sectional area. ${ }^{14}$ Nerve thickening has been demonstrated to be greater among patients with paucibacillary as compared to multibacillary leprosy as well as among those with lepra reaction and longer duration of leprosy as compared to those not in reaction and shorter disease duration. ${ }^{5,15}$ HRUS can also identify focal an-echoic areas indicative of abscesses in the setting of enlarged nerves and aid in carrying out guided aspiration for histological studies, as well as surgical decompression to minimise the extent of nerve damage. While nerve enlargement is emphasised, Chen et al. reported 'atrophy' of nerves, particularly in the lower limbs in subjects with long-standing leprosy. ${ }^{15}$

Ulnar nerve examination showed hypo-echogenicity in 55\% and an oligo-fascicular pattern in $7.1 \%$ in a study by Bathala et al..$^{9}$ In another study, hypoechoic areas and loss of fascicular pattern were noted in $61.4 \%$ and focal hyperechoic areas in $48.9 \% .{ }^{16}$ These features indicate partial or complete loss of fascicular architecture. HRUS observations can be classified as (i) normal, (ii) enlarged nerves with abnormal fascicles, and (iii) enlarged nerves lacking fascicular architecture. ${ }^{17}$ Elias et al. also reported a 'branching' pattern within the hypo-echoic fascicles. ${ }^{8}$ These features mirror the histological changes and can be used to distinguish leprosy from other neuropathies such as entrapment neuropathies. However, this visual grading is subjective and there is a need for studies focussing on quantitative assessment using automatic threshold methods for detecting abnormal nerve echogenicity in patients with leprosy. ${ }^{18}$

Increased vascularity within the nerves can be detected using colour Doppler that reflects intra-neural vascular dilation due to an inflammatory reaction within the nerves during lepra reactions. ${ }^{6,17,19,20}$ Endo-neural vascularity was demonstrated sonographically in $37.5 \%$ of nerves with lepra reaction. ${ }^{16}$ Increased vascularity correlated with cross-sectional area in some studies, but not in others. ${ }^{6,9}$ Increased blood flow may be detected in a small proportion of subjects without thickened nerves and may be an early marker of nerve involvement. ${ }^{6}$ Increased vascularity has been documented in multiple nerves and even in nerves distal from the clinically evident skin lesions in the setting of lepra reactions. This feature may be used to identify peripheral nerves in the state of reaction and pre-empt initiation of steroid therapy even before the onset of symptoms and nerve damage. Post-treatment improvement in hyper-vascularity has been shown to correlate with clinical improvement. On the other hand, persistence of hypervascularity may point towards continued disease activity and therefore the need for continuing treatment for a longer period. ${ }^{17}$

Ultrasound elastography, a technique for identifying elasticity of soft tissues based on the capacity for internal displacement/deformation in response to biomechanical stress, has also been recently applied in leprous neuropathy. The strain ratio of median nerve to flexor digitorum superficialis muscle was distinctly reduced in patients with leprosy as compared to healthy controls, and was lower in those with lepra reaction. ${ }^{21}$ This is a marker of 'stiffer' nerves and may be a useful indicator of fibrosis and thereby as a potential prognostic biomarker.

The size, number and extent of nerves involved in different forms of leprosy, i.e., paucibacillary vs multibacillary and tuberculoid vs lepromatous, reactions and relapses, remain to be studied extensively. Apart from enlarged nerves, hypo-echoic fascicles, loss of fascicular architecture, epineural thickening, edema, increased vascularity and abscesses can be identified by HRUS. While, there exists an established classification for cutaneous manifestations in leprosy that reflects the bacterial load and the immune status of the host, we do not have 
a uniform classification system for leprous neuropathy. Setting up a systematic ultrasound protocol that complements the neurological examination, may open up avenues for a robust scoring system, which would serve not only as a basis for subtyping leprous neuropathy based on the pathology, but also to guide the duration and intensity of treatment and follow-up, as well as for comparison across different cohorts in the setting of drug trials.

\section{References}

1 Raposo MT, Reis MC, Caminha AVQ, Heukelbach J, Parker LA, Pastor-Valero M, Nemes MIB. Grade 2 disabilities in leprosy patients from Brazil: Need for follow-up after completion of multidrug therapy. PLoS Negl Trop Dis, 2018; 12(7): e0006645. doi:10.1371/journal.pntd.0006645.

2 Khadilkar SV, Patil SB, Shetty VP. Neuropathies of leprosy. J Neurol Sci, 2021; 420: 117288. doi:10.1016/j.jn s.2020.117288.

3 Gupte MD, Vallishayee RS, Nagaraju B, Ramalingam A, Lourdusamy G, Kannan S. Inter-observer agreement and clinical diagnosis of leprosy for prophylaxis studies. Indian J Lepr, 1990; 62(3): 281-295. PMID: 2262712.

4 Chen S, Wang Q, Chu T, Zheng M. Inter-observer reliability in assessment of sensation of skin lesion and enlargement of peripheral nerves in leprosy patients. Lepr Rev, 2006; 77(4): 371-376. PMID: 17343224.

5 Suneetha SK, Rao PN. High-resolution ultrasonography in leprosy: value and applications. Indian Dermatol Online J, 2021; 12: 497-499.

6 Jain S, Visser LH, Praveen TL, Rao PN, Surekha T, Ellanti R, Abhishek TL, Nath I. High-resolution sonography: a new technique to detect nerve damage in leprosy. PLoS Negl Trop Dis, 2009; 3(8): e498. doi:10.1371/journal. pntd.0000498.

7 Sreejith K, Sasidharanpillai S, Ajithkumar K, Mani RM, Chathoth AT, Menon PS, George B, Manakkad SP, Neerackal RJ, Menon D, Mann P. High-resolution ultrasound in the assessment of peripheral nerves in leprosy: a comparative cross-sectional study. Indian J Dermatol Venereol Leprol, 2021; 87(2): 199-206. doi:10.25259/ IJDVL_106_20.

8 Elias J Jr, Nogueira-Barbosa MH, Feltrin LT, Furini RB, Foss NT, Marques W Jr, dos Santos AC. Role of ulnar nerve sonography in leprosy neuropathy with electrophysiologic correlation. J Ultrasound Med, 2009; 28(9): 1201-1209. doi:10.7863/jum.2009.28.9.1201.

9 Bathala L, Kumar K, Pathapati R, Jain S, Visser LH. Ulnar neuropathy in Hansen disease: clinical, high-resolution ultrasound and electrophysiologic correlations. J Clin Neurophysiol, 2012; 29(2): 190-193. doi:10.1097/WNP.0b013e31824d969c.

10 Wheat SW, Stryjewska B, Cartwright MS. A hand-held ultrasound device for the assessment of peripheral nerves in leprosy. J Neuroimaging, 2021; 31(1): 76-78. doi:10.1111/jon.12797.

11 Frade MA, Nogueira-Barbosa MH, Lugão HB, Furini RB, Marques Júnior W, Foss NT. New sonographic measures of peripheral nerves: a tool for the diagnosis of peripheral nerve involvement in leprosy. Mem Inst Oswaldo Cruz, 2013; 108(3): 257-262. doi:10.1590/S0074-02762013000300001.

12 Bathala L, N Krishnam V, Kumar HK, Neladimmanahally V, Nagaraju U, Kumar HM, Telleman JA, Visser LH. Extensive sonographic ulnar nerve enlargement above the medial epicondyle is a characteristic sign in Hansen's neuropathy. PLoS Negl Trop Dis, 2017; 11(7): e0005766. doi:10.1371/journal.pntd.0005766.

13 Nagappa M, Pujar GS, Keshavan AH, Bathala L, Jain RD, Das A, Mehndiratta MM, Visser LH, Kumar HM. Sonographic pattern of median nerve enlargement in Hansen's neuropathy. Acta Neurol Scand, 2021; 144(2): 155-160. doi:10.1111/ane.13432.

14 Visser LH, Jain S, Lokesh B, Suneetha S, Subbanna J. Morphological changes of the epineurium in leprosy: a new finding detected by high-resolution sonography. Muscle Nerve, 2012; 46(1): 38-41. doi:10.1002/mus.23 269.

15 Chen X, Zhang L, Huang M, Zhai X, Wen Y, Pan C. Coexistence of nerve enlargement and neuratrophy detected by ultrasonography in leprosy patients. Sci Rep, 2018; 8(1): 7812. doi:10.1038/s41598-018-26085-1.

16 Gupta S, Bhatt S, Bhargava SK, Singal A, Bhargava S. High resolution sonographic examination: a newer technique to study ulnar nerve neuropathy in leprosy. Lepr Rev, 2016; 87(4): 464-475.

17 Martinoli C, Derchi LE, Bertolotto M, Gandolfo N, Bianchi S, Fiallo P, Nunzi E. US and MR imaging of peripheral nerves in leprosy. Skelet Radiol, 2000; 29(3): 142-150. doi:10.1007/s002560050584.

18 Visser LH. Automated threshold techniques for quantitative ultrasound assessment of echogenicity in neuromuscular diseases: A new useful biomarker? Clin Neurophysiol, 2021; 132. doi:10.1016/j.clinph.2021.07.014 
19 Chaduvula MV, Visser LH, Suneetha S, Suneetha L, Devaraju B, Ellanti R, Raju R, Jain S. High-resolution sonography as an additional diagnostic and prognostic tool to monitor disease activity in leprosy: a two-year prospective study. Ultraschall Med, 2018; 39(1): 80-89. doi:10.1055/s-0042-108430.

20 Akita J, Miller LHG, Mello FMC, Barreto JA, Moreira AL, Salgado MH, Kirchner DR, Garbino JA. Comparison between nerve conduction study and high-resolution ultrasonography with color doppler in type 1 and type 2 leprosy reactions. Clin Neurophysiol Pract, 2021; 6: 97-102. doi:10.1016/j.cnp.2021.02.003.

21 Nogueira-Barbosa MH, Lugão HB, Gregio-Júnior E, Crema MD, Kobayashi MTT, Frade MAC, Pavan TZ, Carneiro AAO. Ultrasound elastography assessment of the median nerve in leprosy patients. Muscle Nerve, 2017; 56(3): 393-398. doi:10.1002/mus.25510. 